\title{
ASSESSING COMPETITIVE FITNESS OF IRRADIATED PAINTED APPLE MOTH TEIA ANARTOIDES (LEPIDOPTERA: LYMANTRIIDAE)
}

\author{
D.M. SUCKLING, S.L. WEE and R. PEDLEY \\ HortResearch, PO Box 51, Lincoln \\ Corresponding author: msuckling@hortresearch.co.nz
}

\begin{abstract}
The Australian painted apple moth (Teia anartoides) has been the target of an eradication programme in Auckland. For the first time in New Zealand, the sterile insect technique has been deployed, using males moths irradiated as pupae at $100 \mathrm{~Gy}$. Sterilisation of males has a fitness cost, which was assessed in terms of longevity and competitive fitness. Irradiation at $100 \mathrm{~Gy}$ had no effect on longevity of male painted apple moths. However, when released in separate groups in the flight tunnel, irradiated males were less likely to reach calling females than untreated males $(\mathrm{P}<0.001)$. When single irradiated $(100 \mathrm{~Gy})$ males and untreated males were released together as a pair, irradiated males also showed lower arrival to females $(\mathrm{P}<0.005)$. However, once the males successfully located the females, there were no significant differences between the controls and the irradiated males in the total time spent for mate location, mounting attempts and mating duration.
\end{abstract}

Keywords: painted apple moth, sterile insect technique, irradiation, longevity, fitness.

\section{INTRODUCTION}

The Australian painted apple moth, Teia anartoides (Lepidoptera: Lymantriidae), is the target of an eradication programme in Auckland, using aerial spraying of the insecticidal pathogen Bacillus thuringiensis var. kurstaki (Btk), host removal and the sterile insect technique (SIT) (Suckling 2003). This eradication is easily the largest scale effort ever attempted in New Zealand. The release of sterile male moths is a tactic based on the premise that releases of high numbers of sterile male moths will lead to competitive displacement and reduced reproductive success of wild males.

A preliminary study of irradiated painted apple moth was conducted to support an investigation of dispersal using the mark-release-recapture technique. This showed that the progeny of males irradiated at 160 Gy were sterile by the $\mathrm{F}_{2}$ generation (Suckling et al. 2002). Released moths dispersed up to $5 \mathrm{~km}$ and $17 \%$ of sterile male releases were recaptured. However, recent work has indicated that males dosed at 160 Gy had lower arrival success to calling females in a wind tunnel compared to untreated males (D.M. Suckling, unpubl. data). This may indicate lower field dispersal potential and mating fitness.

A key requirement for the success of SIT is that the fitness of the irradiated males is equivalent to the wild type (IAEA 2000). The application of irradiation should not diminish their longevity or significantly impair the ability of the treated insects to fly, mate and transfer sperm. Quality assurance needs to focus on fitness for purpose, which here overlaps with the evolutionary concept of fitness, expressed as competitive mating ability of treated versus wild-type males.

The lowest effective irradiation dose to achieve sterility is more likely to optimise the mating fitness of the released insects (i.e. maximising the mating with the target population), representing a potential trade-off between sterility and fitness. With Cydia pomonella, the application of a high radiation dose resulted in complete sterility (Bloem et al. 2001). However, the application of a lower irradiation dose gave partially sterile 
insects of superior fitness, which were more effective at population suppression by the second generation post-treatment (Bloem et al. 2001). Previous research has shown the potential of using lower dosages that produce inherited sterility in T. anartoides (Suckling et al. 2002). A 100 Gy treatment gave $80 \%$ sterility of the $F_{1}$ generation and $>99.5 \%$ sterility at $\mathrm{F}_{2}$ level. The few viable individuals from $\mathrm{F}_{1}$ or $\mathrm{F}_{2}$ generation would carry the inherited sterility into the population in which their crossing with the wild-type insects would later lead to eradication of the target population. In addition, recent work by Suckling et al. (2004) showed that administration of $100 \mathrm{~Gy}$ to pupae gave rise to no significant wing deformity (which would potentially affect the flying ability of the adult male) in the treated male painted apple moth. In this paper, the effect of a $100 \mathrm{~Gy}$ dose on the survival and competitive fitness of the irradiated males was investigated.

\section{Insects}

\section{MATERIALS AND METHODS}

Male and female pupae of painted apple moth were received from the HortResearch Mt Albert Research Centre rearing colony in Auckland. The colony was established from field-collected insects, and reared on artificial diet. Upon arrival, the male pupae were kept in a collapsible plastic cage $(11 \times 30 \times 16 \mathrm{~cm})$ lined with tissue while the female pupae were held in disposable Petri dishes $(9 \mathrm{~cm}$ diameter $\times 2 \mathrm{~cm}$ height) for emergence. The insects were provided with moistened dental rolls to maintain humidity within the enclosure.

\section{Irradiation}

Male pupae (6-7 days old) were irradiated using $1.25 \mathrm{MeV}$ gamma rays from a Cobalt ${ }^{60}$ source. Petri dishes ( $9 \mathrm{~cm}$ diameter) containing pupae were positioned on an aluminium scissor jack and periodically rotated to ensure as uniform distribution of dose as possible. The reference point for the irradiations was taken as being at the geometrical centre of the stacked containers, at a distance of $55 \mathrm{~cm}$ from the $\mathrm{Co}^{60}$ source. The entrance air kerma rate (measured free in air, with no back scatter in a $10 \times 10 \mathrm{~cm}$ field at $1 \mathrm{~m}$ ) was $19.9 \mathrm{mGy} / \mathrm{s}$. The radiation field size in the reference plane of the dishes was approximately $11 \times 11 \mathrm{~cm}$. The pupae were irradiated for $5015 \mathrm{~s}$, giving a dose (air kerma) of $100 \mathrm{~Gy}$.

\section{Male longevity}

At $25^{\circ} \mathrm{C}, 26$ untreated and 52 male pupae ( 6 days old) that had been irradiated at 100 Gy were allowed to emerge in a group of ca 10 in separate cages provided with moistened cotton rolls. Upon adult eclosion, survival was observed and recorded daily for 8 days. Chi-square analysis of contingency table was used to compare the frequency of survival between untreated and irradiated males at the eighth day $(\mathrm{P}=0.05)$.

\section{Male flight fitness}

A calling female (1-2 days old) was placed in a small wire-mesh cage ( $4 \mathrm{~cm}$ diameter $\mathrm{x}$ $5 \mathrm{~cm}$ height) with a sticky lid and placed on a sticky base $(18 \times 19 \mathrm{~cm})$ in the upwind area of a perspex flight tunnel $(50 \times 50 \times 150 \mathrm{~cm})$. The flight tunnel was operating at a windspeed of $25-30 \mathrm{~cm} / \mathrm{s}$ under fluorescent light. Air from outside was drawn into the tunnel system through a charcoal filter before passing through a grill, which helped to maintain an even airflow. Temperature of the experimental area was maintained at $25 \pm 2{ }^{\circ} \mathrm{C}$.

Five to ten newly emerged (1-2 days old) irradiated males were released from a plastic tube $(15 \times 21 \mathrm{~cm})$ at the downwind area and their responses to the calling female located $130 \mathrm{~cm}$ upwind were observed for $6 \mathrm{~min}$. Arrival success was defined as when a male moth flew in zigzag anemotaxis and successfully landed on the sticky base within the stipulated time. Numbers of successful arrivals were noted for each replicate. Data obtained were normalized using a modified arcsine (Anscombe 1948) formula:

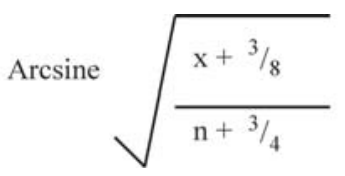


whereby $\mathrm{x}=$ number of males arrived and stuck on the sticky base and $n=$ number of males subjected to flight fitness test in each replicate. Transformed data were then subjected to Student's $t$-test $(\mathrm{P}=0.01)$ for mean comparison. The same procedures were repeated for control males, with 128 replicates for each treatment.

A simple fitness ratio was derived by dividing mean arrival success of irradiated males by that of untreated males.

Male competitive fitness

Irradiated and untreated male pupae were lightly dyed with different fluorescent dyes before adult emergence. A plastic tube containing one irradiated and one untreated male was placed in the downwind area of the flight tunnel. Males were given $5 \mathrm{~min}$ to respond to a calling female that was placed on an inverted Petri dish cover at the upwind end. Within the $5 \mathrm{~min}$ observation period, male behavioural response, i.e. mate location, mounting and mating, was timed. Successfully mated pairs were then removed and kept in a Petri dish and their mating duration was noted. Mating duration was taken from the time a male successfully inserted its aedeagus into female's genitalia until the mating pair separated from each other. Mated pairs were then examined under UV light for dye colour (treatment). A total of 83 replicates were conducted. Successful arrival and mounting attempts of irradiated and untreated males were compared using Chi-Square $\left(\chi^{2}\right)$ goodness of fit test $(\mathrm{P}=0.05)$. Time spent for successful female location, mounting attempts and mating duration were analysed using Student's $t$-test $(\mathrm{P}=0.05)$.

\section{Male longevity}

\section{RESULTS}

There was no mortality recorded for either control or treated males up to the fourth day after emergence (Fig. 1). By 8 days, ca $27 \%$ mortality was recorded for both control and irradiated males, with no evidence of a difference between the treatments at 8 days after emergence $\left(\chi^{2}=0.07 ; \mathrm{P}>0.05\right)$.

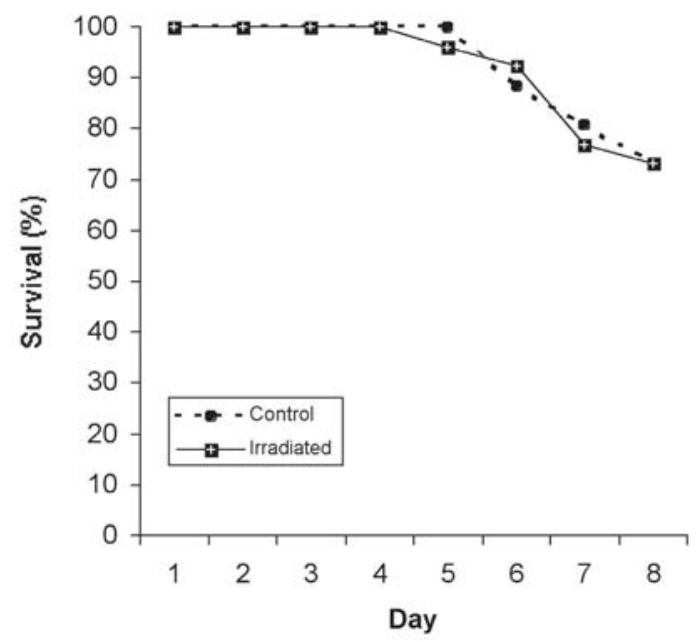

FIGURE 1: Longevity curve of control and irradiated (100 Gy) males of painted apple moth, Teia anartoides, at $25^{\circ} \mathrm{C}$. For the control $\mathrm{n}=26$ and for irradiated moths $\mathbf{n}=\mathbf{5 2}$. 


\section{Male flight fitness}

Both irradiated and control males exhibited similar behavioural responses to the calling females. Responding males initiated wing activation, followed by take-off and flying upwind in zigzag anemotaxis towards the females. At the upwind end, males were stuck on either the sticky base or sticky lid of the cage when they successfully located and arrived at the females. The irradiated males showed a lower arrival success $(27.0 \pm 1.8 \%$; 334 out of 1236 males tested) compared to the untreated controls $(40.9 \pm 1.9 \% ; 504$ out of 1233 males tested) $(\mathrm{t}=5.34, \mathrm{P}<0.001)$. The simple fitness ratio showed that groups of irradiated males were approximately $66 \%$ as successful as groups of untreated males.

\section{Male competitive fitness}

Of the 83 replicates tested with paired releases of irradiated and control males, $37.3 \%$ $(n=31)$ untreated and $12.0 \%(n=10)$ irradiated males successfully landed on the Petri dish containing calling females. The simple fitness ratio showed that the irradiated males were approximately $32 \%$ as successful as the untreated males when they flew as a competitive pair.

Based on these 41 successful replicates, Chi-square analysis showed that the arrival success of untreated males was significantly higher than the irradiated males $\left(\chi^{2}=10.76\right.$, $\mathrm{P}<0.005)$. However, there was no significant difference between the treated and the control males in time spent locating calling females (Table 1).

Of the 31 untreated males that successfully located the females, $25(80.6 \%)$ initiated mating attempts by mounting the females and curling their aedeagus towards the females' genitalia, while 7 out of 10 irradiated males attempted to mount and mate with the calling females. The proportion of irradiated and untreated males that attempted to mate with the calling females within the 5 min observation period was not significantly different. There was also no significant difference in the time spent for mounting activity of the treated and control males (Table 1).

In terms of mating duration, time spent in copula for irradiated males was not significantly different from the control males (Table 1).

TABLE 1: Total time spent in female location, mating attempts and mating duration by the control and irradiated $(100 \mathrm{~Gy})$ males. Values are the mean $\pm \mathrm{SE}$ (min).

\begin{tabular}{lcccc}
\hline & Control & Irradiated & Student's $t$-test & P-value \\
\hline Mate location & $2.33 \pm 0.27$ & $2.01 \pm 0.31$ & 0.631 & $\mathrm{P}>0.05$ \\
Mating attempts & $0.42 \pm 0.06$ & $0.78 \pm 0.38$ & 1.639 & $\mathrm{P}>0.05$ \\
Mating duration & $41.49 \pm 2.62$ & $32.98 \pm 7.79$ & 1.337 & $\mathrm{P}>0.05$ \\
\hline
\end{tabular}

\section{DISCUSSION}

The dose of $100 \mathrm{~Gy}$ irradiation did not affect the survival of male painted apple moth in the first week after emergence, which is vital in the application of the sterile insect technique. In general, sterilising doses do not alter the lifespan of the adults (Snow et al. 1972; Lu et al. 2002). However, in a related study, longevity of irradiated painted apple moth males released in the field or into a gazebo was considerably shorter than in the current laboratory study (D.M. Suckling, unpubl. data).

The results indicated that flight fitness of groups of sterile males treated at $100 \mathrm{~Gy}$ was approximately $66 \%$ as competitive as that of groups of untreated males $(n=1233$ and 1236 for control and treated, respectively). The estimate of the flight fitness of irradiated males tested as a 1:1 competitive pair with untreated males was lower (32\%), although the sample size was also lower ( $n=83$ males of each treatment). Hence it is not clear whether the inferior performance of irradiated males in a competitive situation was really half of that of irradiated males alone. The more representative estimate of flight fitness is likely to be from the larger sample size, given that simultaneous arrival 
of SIT and wild males at a female would be unlikely. Some degree of reduced fitness of the targeted males is inevitable in return for the associated inherited sterility. However, it remains unclear whether the increased amount of fitness from lowered irradiation doses will prove to be 'enough' as a trade-off to lower inherited sterility. Population suppression depends on both mating competitiveness (of treated and $\mathrm{F}_{1}$ males), as well as the survival of potential non-sterile progeny. Modelling of the impact of lower levels of inherited sterility may offer the best approach to optimising this trade-off.

While the irradiated males showed a lower arrival success (ability to seek a female), the results showed that their ability to copulate was not affected. Generally, it is suggested that total sperm transfer has a positive correlation with copulation duration in insects. In Ephestia kuehniella (Lepidoptera: Pyralidae), total time in copula was dose (irradiation) and sperm volume-dependent (i.e. the higher the treatment dose and the lower the sperm number, the longer the total time in copula) (Koudelová \& Cook 2001). Irradiated males tended to copulate for longer times than did untreated controls in gamma irradiated Trichoplusia ni (Holt \& North 1970).

In this study, the irradiated males not only spent as much time in attempting to mate with the untreated females, their mating duration was also not significantly different to that of the untreated males. This suggests that the mating ability/fitness (mounting attempts) and sperm transfer (mating duration) of the irradiated males was not affected at 100 Gy treatments. In another related study by Suckling et al. (2004), female egg production when inseminated by males irradiated at $100 \mathrm{~Gy}$ (as 6-day-old pupae) was not significantly different to that of the control.

This study has used routine wind tunnel flight success as well as more directed experiments on mating success as surrogates for fitness estimates in the field. Irradiated males showed significantly reduced ability to arrive at calling females compared to untreated males. However, upon arrival, irradiated males did not show a lower probability of mating compared to untreated males. This, and the lack of other differences in mating behaviour, suggests that flight success may be an adequate surrogate for the probability of mating of irradiated males, and thereby population introgression of inherited sterility.

It can therefore be concluded that wind tunnel assessment is a valuable tool for quality assurance, and can provide useful insights into the competitive fitness of irradiated males in the implementation of the sterile insect technique.

\section{ACKNOWLEDGMENTS}

This work was funded by MAF Biosecurity Authority and the Foundation for Research, Science and Technology. We would like to thank Anne Barrington for insect supply (Mt Albert Research Centre, Auckland) and John Laban (National Radiation Laboratory) for irradiation services.

\section{REFERENCES}

Anscombe, F.J. 1948: The Transformation of Poisson, Binomial and Negative Binomial Data. Biometrika 35: 246-254.

Bloem, S.; Bloem, K.A.; Carpenter, J.E; Calkins, C.O. 2001: Season-long releases of partially sterile moths for control of codling moth, Cydia pomonella (L.), (Lepidoptera: Tortricidae) in Washington apples. Environ. Entomol. 30: 763-769.

Holt, G.G.; North, D.T. 1970: Effects of gamma irradiation on the mechanisms of sperm transfer in Trichoplusia ni. J. Insect Physiol. 16: 2211-2222.

IAEA 2000: Improvement of Codling Moth SIT to Facilitate Expansion of Field Application. Int. Atomic Energy Agency IAEA-D4-OOCT08578, Vienna, Austria. $29 \mathrm{p}$. 
Koudelová, J.; Cook, P.A. 2001: Effect of gamma radiation and sex-linked recessive lethal mutations on sperm transfer in Ephestia kuehniella (Lepidoptera: Pyralidae). Florida Entomol. 84: 172-182.

Lu, D.G.; Liu, X.H.; Hu, J.G.; Wang, E.D.; He, Q.L.; Li, Y.J. 2002: Cotton bollworm, Helicoverpa armigera (Lepidoptera: Noctuidae): large scale rearing and the effect of gamma radiation on selected life history parameters of this pest in China. In: Proceedings of a final research co-ordination Meeting on Evaluation of Lepidoptera population suppression by radiation induced sterility 1998. Joint FAO/ IAEA Division of Nuclear Techniques in Food and Agriculture. International Atomic Energy Agency, Austria. Pp. 23-27.

Snow, J.W.; Young, J.R.; Lewis, W.J.; Jones, R.L. 1972: Sterilization of adult Fall Armyworms by gamma irradiation and its effect on competitiveness. J. Econ. Entomol. 65: 1431-1433.

Suckling, D.M. 2003: Applying the sterile insect technique for biosecurity: Benefits and constraints. N. Z. Plant Prot. 56: 21-26.

Suckling, D.M.; Hackett, J.; Daly, J. 2002: Sterilisation of painted apple moth Teia anortoides (Lepidoptera: Lymantriidae) by irradiation. N. Z. Plant Prot. 55: 7-11.

Suckling, D.M.; Pedley, R.; Wee, S.L. 2004: Pupal age affects efficacy of irradiation on painted apple moth Teia anartoides. N. Z. Plant Prot. 57: 166-170. 